\title{
Identification and Basic Management of Bleeding Disorders in Adults
}

\author{
Rebecca Kruse-Jarres, MD, Tammuella C. Singleton, MD, and Cindy A. Leissinger, MD
}

Adults with bleeding disorders may present to their family physician with minor bleeding symptoms or hematologic laboratory abnormalities discovered during evaluation for surgery or another purpose. Identifying the small proportion of adults who have an underlying bleeding disorder as the cause for such signs or symptoms may be challenging. In cases of asymptomatic hematologic laboratory abnormalities, the particular abnormality should narrow down the potentially affected hemostatic component(s), ideally streamlining subsequent investigation. In patients presenting with bleeding symptoms, a thorough history and physical examination are critical for first identifying bleeding as pathologic, then performing the appropriate diagnostic evaluation after excluding identifiable causes. Knowledge of the pathophysiologic processes contributing to impaired hemostasis in any given bleeding disorder ensures proper treatment and avoids therapies that are unnecessary or even contraindicated. Management is further determined by bleeding phenotype and, for invasive procedures, the anticipated risk for bleeding. Consultation with a hematologist may facilitate proper evaluation and treatment, particularly in adults with rare bleeding disorders or no identifiable cause for bleeding. This article reviews the diagnostic approach to hematologic laboratory abnormalities and abnormal bleeding in adults, as well as basic preventive care and hemostatic management of adults with bleeding disorders. ( $\mathrm{J}$ Am Board Fam Med 2014;27:549-564.)

Keywords: Blood Coagulation Disorders, Blood Platelet Disorders, Coagulation Protein Disorders, Partial Thromboplastin Time, Prothrombin Time

Adults with undiagnosed bleeding disorders present in a variety of ways. While severe bleeding disorders and most congenital bleeding disorders initially present during childhood, conditions associated with heavy bleeding only after a hemostatic challenge (such as surgery or trauma) often present

This article was externally peer reviewed.

Submitted 2 August 2013; revised 11 April 2014; accepted 21 April 2014.

From the Department of Medicine (RKJ, CAL), and the Department of Pediatrics (TCS), Tulane University School of Medicine, New Orleans, Louisiana.

Funding: Financial support has been provided by Novo Nordisk Inc.

Conflict of interest: RK-J has been a paid consultant for Baxter, Bayer, Biogen Idec, CSL Behring, Grifols, Kedrion, and Novo Nordisk. TCS has been a paid consultant for Baxter, CSL Behring, Grifols, Pfizer, and Novo Nordisk. CAL has been a paid consultant for Bayer, Baxter, Biogen Idec, CSL Behring, Kedrion, Novo Nordisk, and Pfizer.

Corresponding author: Rebecca Kruse-Jarres, MD, Section of Hematology and Medical Oncology, Tulane University School of Medicine, 1430 Tulane Ave., Box SL-78, New Orleans, LA 70112-2699 (E-mail: rkruseja@tulane.edu). during adulthood. This includes milder phenotypes of congenital disorders such as hemophilia and von Willebrand disease (VWD). In addition, acquired disorders such as coagulation factor inhibitors affect adults more often than children. Adults with bleeding disorders may also initially present with abnormal coagulation laboratory studies or seek an initial consultation when a family member is diagnosed with an inherited bleeding disorder. ${ }^{1}$

Given the spectrum of possible presentations, primary care providers may be the first to encounter an adult presenting with an as yet undiagnosed bleeding disorder. Accurate diagnosis depends on an ability to recognize the signs and symptoms that indicate an underlying bleeding disorder, followed by appropriate laboratory testing and interpretation of abnormal coagulation studies. The purpose of this article is to provide practical guidance for the family physician regarding the evaluation of bleeding symptoms and abnormal hematologic laboratory studies in adults. Basic 
preventive care and hemostatic management of those ultimately diagnosed with bleeding disorders also is discussed.

\section{Diagnostic Considerations in an Adult With a Potential Bleeding Disorder Abnormal Bleeding Symptoms}

Bleeding is a common symptom and does not always indicate an underlying bleeding disorder. Symptoms such as gum bleeding, epistaxis, menorrhagia, petechiae, and bruising are especially common; in one study they were reported by anywhere from $22 \%$ to $85 \%$ of men and women without bleeding disorders. ${ }^{2}$ Identification of pathologic bleeding may, therefore, prove challenging. Clinically significant mucocutaneous bleeding is defined as any of the following: spontaneous or provoked bleeding from 2 or more distinct mucocutaneous sites; bleeding from a single site warranting blood transfusions; or bleeding from a single site on 3 or more separate occasions. ${ }^{3,4}$ Bleeding scoring systems have shown promise in retrospectively predicting bleeding phenotype in type $1 \mathrm{VWD}^{5}$ and prospectively excluding mild bleeding disorders in patients presenting with bleeding symptoms or abnormal coagulation study results, ${ }^{6}$ but they require further investigation and validation for broader clinical use.

A thorough history and physical examination often provides clues as to whether bleeding is pathologic and may even point to potential underlying causes. Historical factors to explore are outlined in Table $1 .^{7-11}$ During physical examination, the skin and mucous membranes should be inspected for stigmata of bleeding (eg, bruising, petechiae) and other findings suggestive of potential underlying causes of bleeding (eg, jaundice, telangiectasia). The presence of hepatomegaly, splenomegaly, or joint hypermobility may suggest potential diagnoses associated with bleeding. Skin or conjunctival pallor, tachycardia, or a cardiac flow murmur may indicate associated anemia. Historical and physical findings may suggest an abnormality of either primary hemostasis, which culminates in the formation of a platelet plug; secondary hemostasis, in which fibrin is formed via the coagulation "cascade"; or fibrinolysis, the normal breakdown of clots. A simplified schematic of the coagulation cascade and the corresponding laboratory assays for each pathway are provided in Figure 1. A more
Table 1. Historical Factors to Explore in Adults Presenting With Abnormal Bleeding

Personal history of bleeding and description of bleeding events

Frequency

Severity (including need for any medical or surgical treatments)

Anatomic location

Spontaneous or provoked

Complete medication history, including OTC medications (many of which contain aspirin or NSAIDs)

History of excessive or prolonged bleeding after trauma or surgery (including dental procedures)

History of menorrhagia* (including age of onset) or excessive postpartum bleeding in women

Family history of any of the above bleeding sequelae or of a known heritable bleeding disorder (or consanguinity in cases of autosomal recessive disorders ${ }^{8}$ )

Personal or family history of blood product transfusion and the reasons for transfusion, if known

Personal history or symptoms of any conditions associated with coagulopathy (eg, liver disease) or with specific bleeding disorders (eg, malignancy or autoimmune disease in acquired hemophilia)

History of recurrent miscarriage in women ${ }^{\dagger}$

Personal or family history of thrombotic events ${ }^{\ddagger}$

*Menorrhagia is defined by heavy menstrual bleeding of more than 7 days' duration ${ }^{7} ;>80 \mathrm{~mL}$ of blood loss per menstrual cycle $^{7}$; needing to change sanitary pads or tampons more than hourly ${ }^{11}$; or passage of clots $>1.1$ inch in diameter. ${ }^{11}$

${ }^{\dagger}$ Recurrent miscarriage may be a feature of some congenital factor (eg, factor XIII) and fibrinogen deficiencies. ${ }^{10}$

${ }^{\ddagger}$ Arterial or venous thromboses may be a feature of some congenital factor (eg, factor XI, factor VII, fibrinogen, factor V, and factor XIII) deficiencies. ${ }^{9}$

NSAID, nonsteroidal anti-inflammatory drug; OTC, over the counter.

thorough review of the complex process of hemostasis and its various components is beyond the scope of this article but can be found elsewhere. ${ }^{12-14}$

Excessive bruising, epistaxis, bleeding after dental extraction, and menorrhagia are symptoms suggestive of quantitative or qualitative platelet disorders. ${ }^{8} \mathrm{~Pa}$ tients with platelet abnormalities may also experience excessive bleeding after hemostatic challenges. ${ }^{15}$ The presence of petechiae in particular suggests a platelet defect. Quantitative platelet abnormalities in adults are most often acquired; autoimmune (ie, idiopathic thrombocytopenic purpura [ITP]) and drug-induced thrombocytopenia account for the vast majority of cases of isolated thrombocytopenia. ${ }^{16}$ Certain acquired thrombocytopenic conditions (eg, disseminated intravascular coagulation [DIC] and thrombotic thrombocytopenic purpura $[\mathrm{TTP}] /$ hemolytic 
Figure 1. ${ }^{12,29}$ Simplified schematic of the coagulation "cascade." The coagulation cascade consists of three pathways: the intrinsic pathway, the extrinsic pathway, and the final common pathway, culminating in the formation of fibrin. This model of coagulation oversimplifies the process of in vivo coagulation but is useful for the correlation of coagulation assay (ie, activated partial thromboplastin time [aPTT], prothrombin time [PT]) abnormalities with specific pathways and, hence, coagulation factors. FI, fibrinogen; FII, factor II; FIX, factor IX; FV, factor V; FVII, factor VII; FVIII, factor VIII; FX, factor X; FXI, factor XI; FXII, factor II; HMWK, high-molecularweight kininogen; PK, prekallikrein; TF, tissue factor.

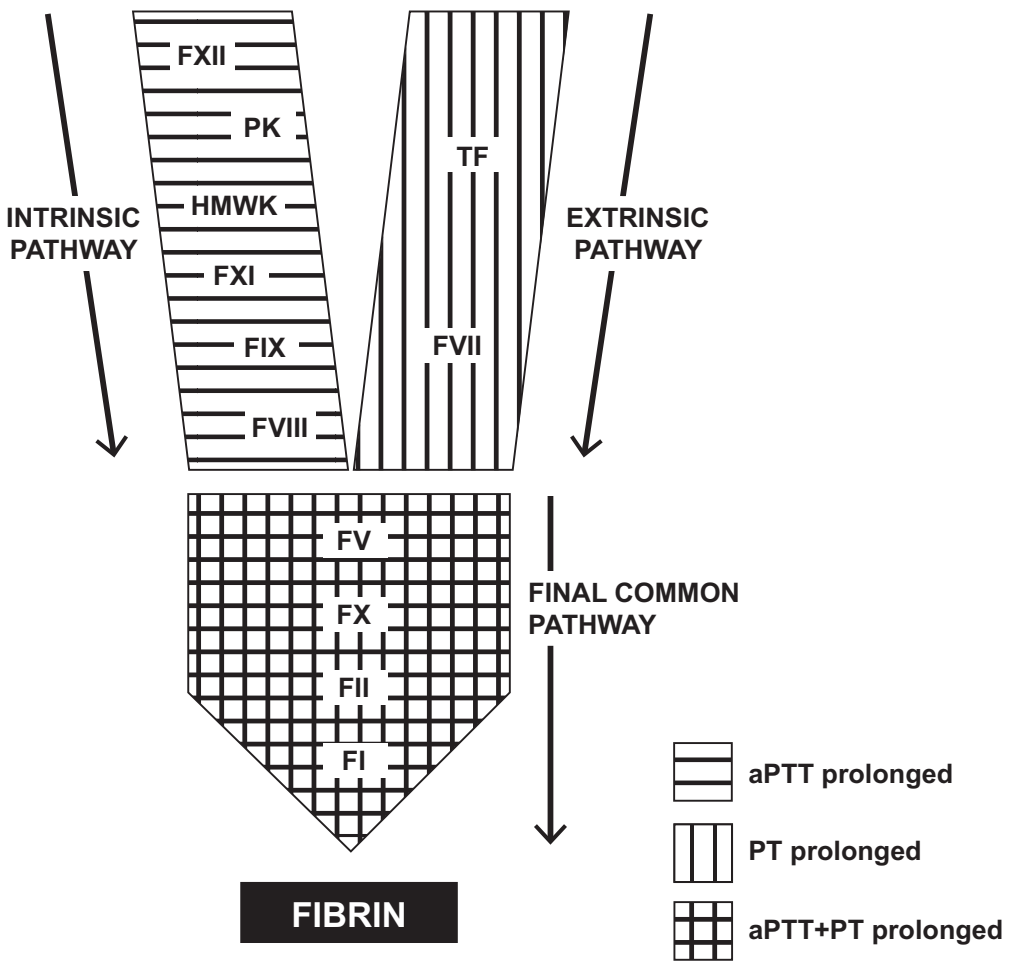

uremic syndrome) may present with bleeding in acutely ill patients (Table 2) but may also be the underlying reason for asymptomatic thrombocytopenia in an ambulatory patient. Congenital (or inherited) thrombocytopenias are usually diagnosed during childhood, but diagnosis could be delayed into adulthood, especially in individuals who do not regularly obtain health care. Numerous inherited thrombocytopenias exist; individual conditions may be identified based on platelet size, coexisting physical or laboratory abnormalities, and the presence of defective platelet function as well as an abnormal platelet count. ${ }^{8,17,18}$ In most inherited forms, thrombocytopenia is mild: bleeding occurs only occasionally or after hemostatic challenge. ${ }^{17} \mathrm{~A}$ family history of thrombocytopenia (including thrombocytopenia erroneously attributed to other causes such as $\mathrm{ITP}^{16}$ ) may suggest an inherited thrombocytopenia. Conversely, documentation of previously normal platelet counts would exclude congenital thrombocytopenia. ${ }^{19}$
Qualitative platelet disorders presenting in adults can be caused by medication (eg, aspirin and nonsteroidal anti-inflammatory drugs [NSAIDs]), uremia, cirrhosis, and myeloproliferative disorders. $^{3,20}$ Several inherited disorders of platelet function exist as well and are classified in various ways (by defective platelet function [eg, adhesion or aggregation] or platelet component [eg, receptors, granules, or membrane phospholipids]). ${ }^{8,15}$ Although severe inherited disorders of platelet receptors (eg, Glanzmann thrombasthenia and Bernard-Soulier syndrome) and some platelet granule disorders typically present earlier in life, the majority of inherited platelet function disorders present during adulthood, often after hemostatic challenge. . $^{81}$

VWD may also be considered a disorder of platelet function, given the role of von Willebrand factor (VWF) in platelet adhesion and aggregation. ${ }^{22}$ Consequently, VWD tends to present with clinical signs and symptoms similar to those of 
Table 2. Differential Diagnosis and Diagnostic Features of Quantitative Platelet Disorders

\begin{tabular}{|c|c|c|}
\hline $\begin{array}{l}\text { Mechanism of } \\
\text { Thrombocytopenia }\end{array}$ & Differential Diagnosis & Comments \\
\hline \multirow[t]{9}{*}{ Impaired production } & Hematologic malignancies ${ }^{20}$ & $\begin{array}{l}\text { Often accompanied by abnormalities in other marrow cell } \\
\text { lines (ie, red and white blood cells) }\end{array}$ \\
\hline & Aplastic anemia ${ }^{41}$ & Other marrow cell lines affected as well \\
\hline & Myelodysplasia $^{20}$ & $\begin{array}{l}\text { May be accompanied by abnormalities in other marrow cell } \\
\text { lines; bleeding may occur at higher platelet counts than } \\
\text { expected }\end{array}$ \\
\hline & Drugs/toxins ${ }^{41,42}$ & For example, alcohol, chemotherapeutic agents, radiation \\
\hline & Viral marrow suppression or damage ${ }^{42}$ & For example, because of EBV, parvovirus, $\mathrm{HCV}$, or HIV \\
\hline & Gestational thrombocytopenia $^{43}$ & $\begin{array}{l}\text { Generally mild [ie, platelet count }>70,000 / \mathrm{mL} \text { ] and self- } \\
\text { limited, resolving after delivery; exact mechanism of } \\
\text { thrombocytopenia unknown-hemodilution and } \\
\text { increased platelet turnover may also contribute }\end{array}$ \\
\hline & Liver disease ${ }^{42}$ & $\begin{array}{l}\text { Because of reduced levels of thrombopoietin, which is } \\
\text { produced by the liver }\end{array}$ \\
\hline & Nutritional deficiencies $^{42}$ & For example, folate, vitamin $\mathrm{B}_{12}$ \\
\hline & Inherited thrombocytopenias ${ }^{16,17,19,41}$ & $\begin{array}{l}\text { Often present with incidental thrombocytopenia in } \\
\text { adulthood; may have family history of thrombocytopenia } \\
\text { or personal history of low platelet counts }\end{array}$ \\
\hline
\end{tabular}

Destruction or consumption Immune
Medication, including heparin (most common), various antimicrobial, antiarrhythmic, anticonvulsant, and antifungal agents, and $\mathrm{H} 2$ receptor antagonists $^{41}$

ITP

\author{
Autoimmune disease $\mathrm{H}^{41}$ \\ Infection $^{41,45}$ \\ TTP in presence of ADAMTS13 \\ autoantibodies ${ }^{41,46}$
}

Nonimmune

HUS

$\mathrm{DIC}^{41}$

Sepsis $^{42}$

HELLP syndrome ${ }^{44}$

Physical destruction ${ }^{41,42}$

Sequestration

Splenomegaly ${ }^{41,42}$
Along with drug-induced thrombocytopenia, accounts for majority of isolated thrombocytopenia in adults ${ }^{16}$; typically chronic in adults ${ }^{41}$; may occasionally be accompanied by Coombs positive hemolytic anemia [Evans syndrome $]^{41}$

For example, HIV

Coombs-negative hemolytic anemia and thrombocytopenia; may or may not have associated renal insufficiency, fever, and mental status changes; neurological symptoms vary, ranging from headache and confusion to seizures and stroke-like symptoms

Relatively uncommon but life-threatening cause of thrombocytopenia; classic form consists of microangiopathic hemolytic anemia, thrombocytopenia, and renal failure

Other hallmark laboratory findings include decreased fibrinogen, elevated fibrin degradation products, and a positive D-dimer

Serious intrapartum condition characterized by hemolysis and elevated liver enzymes in addition to thrombocytopenia; frequently coexists with preeclampsia; may recur in subsequent pregnancies

For example, valvular disease, cardiopulmonary bypass, cavernous hemangiomas (eg, in Kassabach-Merritt syndrome)

ADAMTS, a disintegrin and metalloproteinase with thrombospondin type 1 motives; DIC, disseminated intravascular coagulation; EBV, Epstein-Barr virus; HCV, hepatitis C virus; HELLP, hemolysis, elevated liver enzymes, and low platelets; HIV, human immunodeficiency virus; HUS, hemolytic uremic syndrome; ITP, idiopathic thrombocytopenic purpura; TTP, thrombotic thrombocytopenic purpura. 
platelet disorders. In patients with a relevant history (eg, significant mucocutaneous bleeding, family history), initial hematologic laboratory evaluation should include specific testing for VWD (VWF antigen, VWF ristocetin cofactor activity, and factor VIII activity assays); other screening tests such as activated partial thromboplastin time (aPTT) and bleeding time or a platelet function analyzer (PFA-100; Siemens Health care Diagnostics Inc., Tarrytown, NY) may miss VWD. ${ }^{23}$ Routine hemostatic screening laboratories may likewise miss other platelet function defects. Consequently, in patients with mucocutaneous bleeding who do not have thrombocytopenia or VWD, platelet aggregometry should be considered as an initial test for assessing platelet function, ${ }^{3}$ in addition to evaluation of a peripheral blood smear for abnormalities in platelet morphology that are specific to certain conditions (eg, gray platelet syndrome).

In contrast to the superficial bleeding associated with platelet defects, coagulation factor defects result in delayed, deep bleeding, for example, into muscles or joints, as well as deep soft-tissue and mucocutaneous bleeding. Patients with milder congenital deficiencies or those with certain specific congenital deficiencies (eg, factor XI deficiency) are more likely to bleed after hemostatic challenge.

Menorrhagia is a common bleeding symptom in women, both those with and without bleeding disorders. Menorrhagia is the most common bleeding symptom in women with inherited bleeding disorders, ${ }^{24}$ particularly menorrhagia that begins at menarche and persists into adulthood. ${ }^{25}$ When menorrhagia begins after the age of 20 years, acquired bleeding diatheses should be considered, as should nonhematologic causes such as uterine pathology (eg, fibroids), hypothyroidism, and, in women older than 40 years of age, perimenopausal anovulation. ${ }^{25}$ Consensus recommendations for the evaluation of acute menorrhagia have been published. ${ }^{25}$ They include assessments of the patient's menstrual, bleeding, medication, and family histories; speculum and pelvic examinations with subsequent Papanicolaou test and endometrial biopsy, as appropriate, based on such factors as patient age and feasibility of performing these interventions through heavy menstrual bleeding; and (preferably intravaginal) ultrasound. ${ }^{25}$ Recommended initial laboratory testing includes a complete blood count, pregnancy test, prothrombin time (PT), aPTT, fibrinogen, and, if feasible, VWF levels. ${ }^{25}$ Additional studies may include tests of liver or platelet function or specific factor levels, as clinically indicated. Samples may also be drawn for storage for future testing, ${ }^{25}$ especially if administration of transfusional therapies (eg, fresh frozen plasma [FFP]) is anticipated.

\section{Hematologic Laboratory Abnormalities}

Once a significant bleeding history is identified, an initial laboratory evaluation is generally undertaken to determine the underlying cause. Alternatively, an adult with an undiagnosed bleeding disorder may present with abnormal hematologic laboratory studies obtained as part of an evaluation for surgery or for some other reason. Increased sensitivity of the reagents used in coagulation assays, most notably PT and aPTT, has led to an increased incidence in abnormal results for these tests. ${ }^{26}$ Screening coagulation laboratory studies have a low yield overall in the absence of any symptoms or family history of an underlying bleeding disorder. ${ }^{27}$ Even among patients with a high pretest probability of having a bleeding disorder, only a minority of abnormalities correspond with a clinically significant bleeding diathesis. ${ }^{28}$ Failure to identify the subset of individuals whose abnormal coagulation studies signify an as yet undiagnosed bleeding disorder, however, may have serious, if not grave, consequences, particularly if an invasive intervention is planned.

\section{Abnormal Coagulation Assays}

Prolongation of the aPTT or PT may indicate an acquired or congenital clotting factor deficiency or an inhibitor of one or more coagulation factors. Potential inhibitors include medication (namely anticoagulants), antibodies directed against specific coagulation factors, and nonspecific inhibitors (eg, lupus anticoagulants). A mixing study can be used to differentiate a deficiency from an inhibitor. In a mixing study, equal volumes of normal and patient plasma are combined, and then the coagulation study is repeated. In cases of coagulation factor deficiency, the presence of normal plasma replaces the missing factor(s), thereby normalizing the abnormal coagulation study. In contrast, when an inhibitor is present the abnormality persists after the addition of normal plasma. In some cases, a prolonged incubation period after mixing is necessary for accurate interpretation of results; therefore, it is imperative that the mixing study be incubated at $37^{\circ} \mathrm{C}$ for 2 hours. Readily identifiable 
Figure 2. ${ }^{29-32}$ Differential diagnosis for abnormalities of aPTT and PT. Once the coagulation laboratory study abnormality has been identified, the differential diagnosis may be further narrowed down based on the specific coagulation study abnormalities (activated partial thromboplastin time [aPTT], prothrombin time [PT], or both); the presence or absence of bleeding symptoms; and the results of the mixing study. Note that prolonged incubation may be required for accurate mixing study results. *PT may also be prolonged by heparin (at high doses) or direct thrombin inhibitor (DTIs). ${ }^{\dagger}$ aPTT may also be prolonged in FII and FX deficiencies. ${ }^{*}$ aPTT may also be prolonged in the setting of advanced liver disease or vitamin $\mathrm{K}$ deficiency. ${ }^{\S}$ Applies to $10 \%$ of lupus anticoagulants. DIC, disseminated intravascular coagulation; FII, factor II; FIX, factor IX; FV, factor V; FVII, factor VII; FVIII, factor VIII; FX, factor X; FXI, factor XI; FXII, factor XII; HMWK, high-molecular-weight kininogen; VWD, von Willebrand disease.

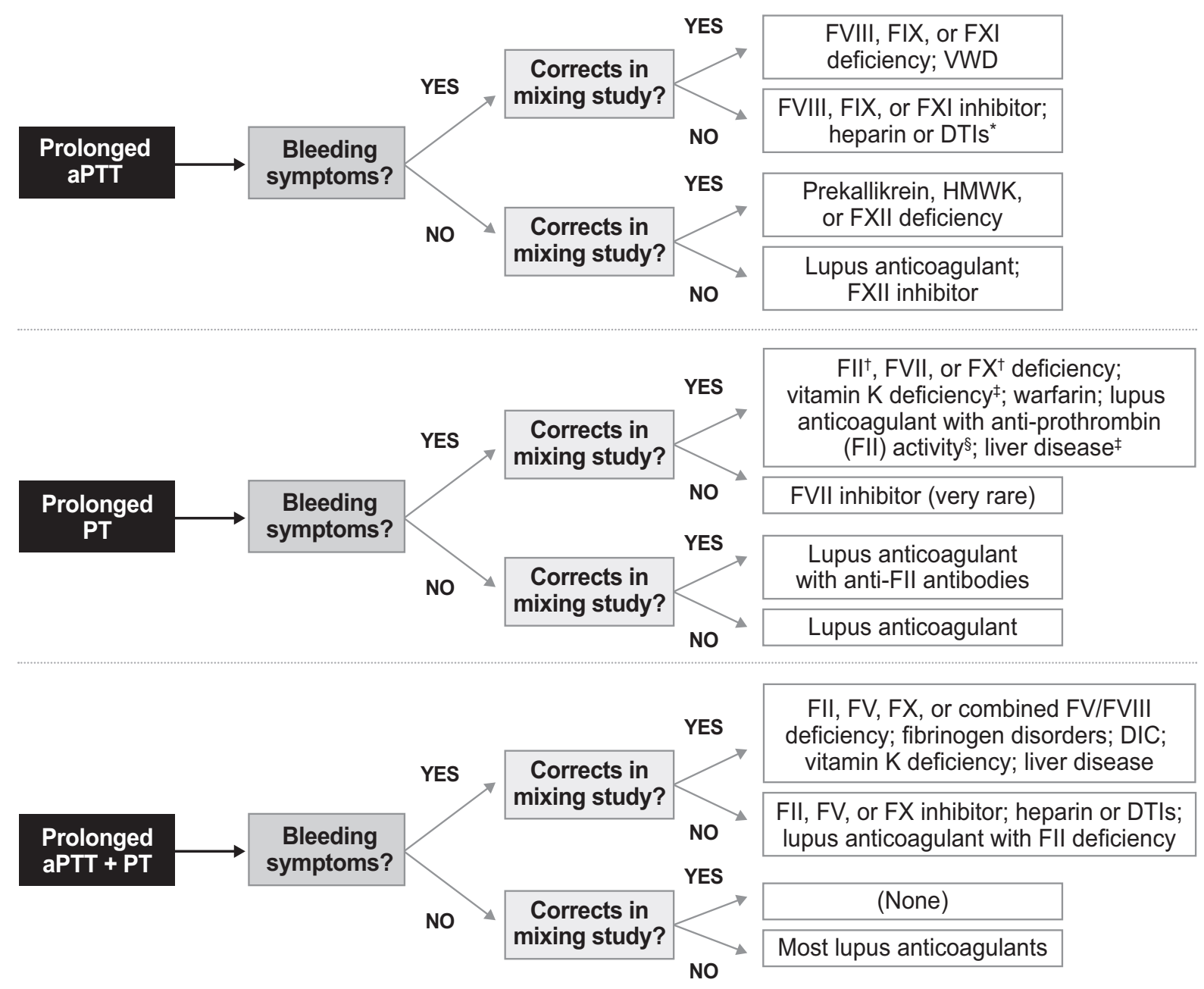

causes of coagulation study abnormalities (eg, anticoagulant medication, systemic diseases such as liver disease, or artifactual prolongation [eg, caused by sample "contamination" with heparin]) should ideally be excluded before proceeding to a mixing study. ${ }^{29}$

The potential coagulation factors involved and, therefore, possible diagnoses can be narrowed down based on which coagulation study is abnor$\mathrm{mal}^{29-32}$ (Figures 1 and 2). Isolated prolongation of the aPTT indicates an abnormality of the intrinsic pathway (ie, of prekallikrein, high-molecular-weight kininogen, factor VIII [FVIII], factor IX, factor XI, or factor XII [FXII])..$^{29,30}$ An isolated prolonged aPTT can also indicate a lupus anticoagulant. An isolated prolonged PT indicates an abnormality of the extrinsic pathway (ie, factor VII [FVII]). ${ }^{29,30}$ On occasion, congenital deficiencies of the final common pathway factors II (FII, also known as prothrombin), $\mathrm{V}(\mathrm{FV})$, and $\mathrm{X}(\mathrm{FX})$ and fibrinogen 
present with an isolated prolonged PT and a normal aPTT. ${ }^{29}$ Not all conditions that prolong aPTT or PT are associated with a bleeding phenotype (Figure 2). For example, lupus anticoagulant is more likely to be associated with thrombosis than with bleeding, except in rare cases of associated antiprothrombin antibodies, which lead to bleeding symptoms and a prolonged PT in addition to prolonged aPTT. ${ }^{32}$ Deficiencies of the contact-activating factors (FXII, prekallikrein, and high-molecular-weight kininogen) are rare and do not cause easy bleeding; however, they are associated with markedly prolonged aPTTs. When asked to evaluate an asymptomatic patient who has a markedly prolonged aPTT, testing for lupus anticoagulant should be considered first when the mixing study fails to correct and for FXII deficiency when the aPTT corrects in the mixing study.

Prolongation of both aPTT and PT isolates the abnormality to the final common pathway, consisting of FV, FX, FII, and fibrinogen. ${ }^{29,30}$ Congenital or acquired deficiencies of any of these factors may present with a prolonged PT and aPTT. Acquired deficiencies of single coagulation factors may occur in the setting of systemic diseases such as amyloidosis (FX) and myeloproliferative disease (FV) and must be differentiated from congenital deficiencies. $^{29}$ Deficiencies of multiple factors from both the intrinsic and extrinsic pathways or from all 3 pathways may also simultaneously prolong aPTT and PT. ${ }^{29}$ Multiple factor deficiencies may occur as a result of severe liver disease, supratherapeutic warfarin doses resulting in deficiency of vitamin $\mathrm{K}$-dependent factors, or consumptive coagulopathy (ie, DIC, which generally occurs in the setting of systemic illness and is therefore unlikely to present solely with asymptomatic coagulation study abnormalities). Potential inhibitors that may present with prolonged aPTT and PT include heparin, direct thrombin inhibitors, potent lupus anticoagulants, and other nonspecific inhibitors such as those associated with lymphoproliferative disorders or monoclonal protein disorders. Bleeding symptoms are generally a feature of all conditions that simultaneously prolong PT and aPTT.

Vitamin K deficiency and liver disease both may result in prolongation of $\mathrm{PT}$ or, in more advanced stages, of both PT and aPTT. The vitamin K-dependent coagulation factors (II, VII, IX, and X) may become depleted because of malabsorption, prolonged antibiotic use, or warfarin therapy. ${ }^{29}$
Liver disease is distinguished from vitamin $\mathrm{K}$ deficiency by a deficiency of $\mathrm{FV}$ in addition to the vitamin $\mathrm{K}$-dependent factors. ${ }^{29}$ Liver disease is often quite advanced by the time abnormalities in coagulation laboratory studies are present; therefore, patients with liver disease are unlikely to present solely with asymptomatic coagulation laboratory abnormalities and often have concurrent physical signs (eg, jaundice, hepatomegaly) or other laboratory abnormalities indicative of impaired hepatic function (eg, thrombocytopenia, hypoalbuminemia, transaminitis).

Acquired coagulation factor inhibitors (or autoantibodies), most commonly directed against FVIII (a condition referred to as acquired hemophilia), deserve special mention because they may be associated with serious bleeding in adults with no history of bleeding. Acquired hemophilia is a rare condition (incidence of 1 to 4 per million per year ${ }^{33}$ ) that predominately affects older adults. In the largest collection of affected patients to date $(\mathrm{n}=501)$, the median age at diagnosis was 74 years; however, younger women in particular may be affected as well because of an association with pregnancy. ${ }^{34}$ In approximately half of cases, a coexisting underlying condition such as pregnancy is identified, ${ }^{35}$ some of which (eg, cancer, autoimmune disease) are characterized by immune dysregulation. Acquired hemophilia should be suspected in an adult with new- or recent-onset bleeding who has no personal or family history of bleeding and presents with an isolated prolonged aPTT that does not correct in a mixing study. ${ }^{35}$ Acquired hemophilia was associated with an especially high mortality - up to $41 \%$ in untreated patients ${ }^{36-38}$ and $6 \%$ to $8 \%$ among effectively treated patients, ${ }^{39,40}$-mostly because of rebleeding. Because acquired hemophilia requires specialized treatment, prompt diagnosis is important, particularly when an invasive procedure is necessary. ${ }^{38}$

\section{Thrombocytopenia}

Thrombocytopenia is defined as a platelet count below the lower limit of the normal range (ie, $<150,000 / \mu \mathrm{L}$ in most laboratories). In asymptomatic patients, artifactual thrombocytopenia as a result of platelet clumping may first be excluded by examining the peripheral blood smear. ${ }^{41}$ Thrombocytopenia occurs because of impaired production, ${ }^{16,17,19,20,41-43}$ destruction or consumption, ${ }^{16,41,42,44-46}$ or sequestration ${ }^{41,42}$ of platelets 
Table 3. Bleeding Disorders and Associated Conditions Presenting With Normal Initial Hematologic Laboratory Study Results

\begin{tabular}{ll}
\hline Condition & Recommended Screening Tests \\
\hline VWD $^{23 *}$ & VWF antigen, VWF ristocetin cofactor activity, and FVIII activity assays \\
FXIII deficiency $^{59}$ & $\begin{array}{c}\text { Quantitative functional FXIII assay (ammonia-release or amine-incorporation } \\
\text { assay) }\end{array}$ \\
$\begin{array}{c}\text { Fibrinolytic disorders }(\alpha 2 \text {-antiplasmin and PAI-1 } \\
\text { deficiencies) }\end{array}$ & $\begin{array}{c}\text { Specific functional (activity) and antigen assays } \\
\text { Collagen disorders }(\mathrm{eg}, \mathrm{EDS}, \mathrm{BJHS})^{58}\end{array}$ \\
\hline
\end{tabular}

*Initial hematologic laboratory studies will be normal in only some cases of von Willebrand disease (VWD). Nevertheless, additional screening tests should be performed before excluding VWD based on a normal activated partial thromboplastin time or Platelet Function Analyzer, when clinically indicated.

BJHS, benign joint hypermobility syndrome; EDS, Ehlers-Danlos syndrome; FXIII, factor XIII; FVIII, factor VIII; PAI, plasminogen activator inhibitor; VWF, von Willebrand factor.

(Table 2). Immune thrombocytopenias, either idiopathic (primary) or secondary to an autoimmune disease, may present with asymptomatic, isolated thrombocytopenia. ${ }^{47}$ Other potential causes in ambulatory patients include medication; infectious agents such as Epstein-Barr, human immunodeficiency, or hepatitis $\mathrm{C}$ virus; or primary marrow failure. ${ }^{41}$ Conversely, certain thrombocytopenic conditions may be excluded based on the lack of specific predisposing factors or acute illness (eg, shiga toxin-induced hemolytic uremic syndrome or DIC). Bleeding propensity in thrombocytopenic conditions usually depends on the platelet count. Bleeding is generally mild and limited to easy bruising when platelet counts exceed $20,000 / \mu \mathrm{L}^{41}$ The risk for spontaneous bleeding increases only after the platelet count decreases to $<10,000 / \mu \mathrm{L},{ }^{41}$ except in ITP, in which the increased presence of young, hyperfunctional platelets may preserve hemostasis even at platelet counts below this level. ${ }^{48}$

\section{Abnormal Platelet Function}

Historically, bleeding time was used as a screening test for qualitative platelet abnormalities, including in patients undergoing invasive procedures, particularly those who were recently exposed to medication that might alter platelet function (eg, aspirin or NSAIDs). This test also was used to screen for certain bleeding disorders, including VWD. ${ }^{23,29}$ However, bleeding time has been shown to be relatively insensitive and poorly reproducible. $^{23,49,50}$ The test has not been shown to predict excessive surgical bleeding (particularly without other history suggesting a bleeding disorder) or to reliably identify aspirin- or NSAID-induced plate- let dysfunction. ${ }^{49}$ In addition, some patients with VWD have a normal bleeding time. ${ }^{23}$ Therefore, this test is no longer routinely recommended as part of an initial hemostatic laboratory evaluation, especially to screen for VWD. ${ }^{23}$ For assessing platelet function, the PFA-100 has supplanted the bleeding time in many laboratories. ${ }^{51}$ In this technique, a sample of whole blood is passed through an aperture in a membrane coated with collagen and either epinephrine or adenosine diphosphate, and the amount of time it takes for the membrane to become occluded (ie, the closure time) is measured. ${ }^{51}$ A closure time exceeding 300 seconds is considered prolonged. ${ }^{52}$ Although the PFA-100 has been shown to have a relatively high sensitivity for detecting moderate and severe VWD, aspirinand NSAID-related platelet dysfunction, and severe platelet function disorders, closure times may be normal in milder VWD and platelet function disorders, including relatively common platelet storage pool deficiencies, ${ }^{51-55}$ thereby limiting the usefulness of the PFA-100 as a screening tool for all platelet function disorders. ${ }^{54}$ If a patient is suspected of having a significant platelet function disorder, consultation with a hematologist is suggested to obtain more extensive platelet aggregation and release studies in a specialized laboratory setting.

\section{Normal Initial Hematologic Laboratory Studies}

Of note, several bleeding disorders are associated with normal routine initial hematologic laboratory studies (ie, platelet count, PT, aPTT, and PFA100) (Table 3). In some cases, the sensitivity of these studies for detecting certain conditions, such 
as some types of VWD or milder factor deficiencies, may be limited. In other cases, neither fibrin generation nor platelet function is impaired; therefore, coagulation studies and quantitative and qualitative platelet test results are normal. Alternative screening tests are indicated when these bleeding disorders are suspected based on clinical grounds ${ }^{13,23,56-60}$ (Table 3).

\section{Therapeutic Strategies in Patients With Bleeding Disorders \\ Preventive Care and Measures}

Some basic guidelines apply for all patients with bleeding disorders in the primary care setting. In general, any medication that may impair hemostatic function, such as aspirin and NSAIDs, should be avoided, particularly during bleeding episodes. Proper preventive care, including screening for common age-related comorbidities and associated risk factors, is important in this population. Certain conditions may be particularly problematic in patients with bleeding disorders; for example, untreated severe hypertension may lead to intracranial bleeding. ${ }^{61}$ Invasive preventive or screening procedures should not necessarily be deferred because of the risk for bleeding. Routine dental care may be especially important in patients with bleeding disorders to avoid the need for more extensive dental procedures down the line. ${ }^{62,63}$ Invasive screening procedures such as colonoscopy may be performed with minimal bleeding risk. ${ }^{64}$ Consultation with a hematologist should be considered before such invasive procedures to determine whether systemic hemostatic coverage is indicated and, if so, what this coverage should consist of. Hemostatic therapy often is not needed for minimally invasive procedures, particularly if no tissue incision or excision is required. ${ }^{64}$ Meticulous surgical technique and topical measures such as suturing and use of fibrin glue, oxidized cellulose (eg, Surgicel [Ethicon, LLC, San Lorenzo, Puerto Rico]), or topical antifibrinolytics (eg, tranexamic acid) may be sufficient to maintain hemostasis or at least minimize the use of systemic hemostatic treatments in some dental procedures. ${ }^{65-67}$ Such topical measures may also be used for bleeding from wounds, in addition to closing (when appropriate), applying pressure to, or packing the wound. Packing can also be used to control epistaxis. ${ }^{68}$ With diligent attention to local hemostasis, systemic treatments can often be avoided in cases of minor bleeding.

\section{Hemostatic Therapies}

Consultation with a hematologist is strongly recommended before initiating systemic therapies to discuss treatment options and to ensure that samples for all indicated laboratory studies are collected before any blood products are administered, since this may affect the reliability of any results. Transfusion of the missing or defective hemostatic component may not be indicated or effective in all cases. For example, transfusion of platelets may be ineffective in ITP and, in the absence of life-threatening hemorrhage, is contraindicated in microangiopathic conditions such as TTP and in heparininduced thrombocytopenia, given the risk for exacerbating these conditions and fueling thrombosis. ${ }^{45,69,70}$ Similarly, in the setting of liver disease, infusion of large volumes of FFP to treat presumptive coagulopathy may exacerbate bleeding that is a result of elevated venous pressure (eg, variceal bleeding) by further increasing this pressure. ${ }^{71}$ In addition, factor replacement may be ineffective in factor deficiencies because of an acquired inhibitor (antibody against the clotting factor concentrate). Other specialized treatments or interventions may be indicated or advised for immediate management of specific bleeding disorders (eg, plasma exchange for TTP or immunomodulatory therapies for immune-mediated thrombocytopenia).

\section{Platelet Transfusion}

Indications for platelet transfusion in patients with thrombocytopenia vary based on the underlying mechanism of thrombocytopenia. ${ }^{41,69,70}$ Thrombocytopenia as a result of reduced production is an indication for platelet transfusion, and the thresholds for transfusion vary based on the indication (Table 4). There are no such thresholds for platelet transfusion in patients with immune-mediated thrombocytopenia or platelet function defects. ${ }^{69}$ Platelet transfusion should only be undertaken in patients with autoimmune thrombocytopenia in instances of serious or life-threatening bleeding (eg, gastrointestinal or intracranial hemorrhage). ${ }^{69,70}$ Large amounts of platelets may be required to boost the platelet count in the setting of autoimmune thrombocytopenia, given the shortened survival of the transfused platelets; concomitant ad- 
Table 4. Indications for Platelet Transfusion ${ }^{41,69,70}$

Thrombocytopenia due to impaired
platelet production

Autoimmune thrombocytopenia

Platelet function disorders
Platelet count thresholds for platelet transfusion

Prophylactic transfusion: $<10,000 / \mu L^{*}$

Coverage for invasive procedures

Minor invasive procedures (eg, LP, CVC placement, epidural anesthesia, endoscopy with biopsy, or liver biopsy): 50,000/ $\mu \mathrm{L}^{\dagger}$

Major surgery in noncritical sites: $50,000-100,000 / \mu \mathrm{L}^{\ddagger}$

Major surgery in critical sites (eg, eyes, brain): 100,000/ $\mu \mathrm{L}$

Active bleeding: $50,000-100,000 / \mu \mathrm{L}$

Platelet transfusion should only be undertaken in instances of serious or lifethreatening bleeding (eg, gastrointestinal or intracranial hemorrhage)

Large amounts of platelets may be required to boost the platelet count, given the shortened survival of the transfused platelets; concomitant administration of immune-modulating therapies (eg, steroids or IVIG) may attenuate this process, resulting in a more rapid rise in platelet count

For active bleeding or hemostatic surgical coverage

Eliminate or mitigate any factors contributing to platelet dysfunction (eg, medications, underlying conditions)

Desmopressin may be considered in patients with inherited platelet function disorders, particularly storage pool defects, or in patients with uremia

Platelet transfusion may be considered when the above measures are not indicated or are unsuccessful

\footnotetext{
*In some cases, the platelet count threshold for prophylactic transfusion may be higher (eg, in patients with sepsis, acute bleeding, other coagulation abnormalities, or necrotic tumors receiving aggressive treatment) or lower (eg, in patients at risk for platelet alloimmunization or refractoriness because of frequent transfusions). ${ }^{69,70}$

${ }^{\dagger}$ The threshold for platelet transfusion before bone marrow aspiration and biopsy is unknown. ${ }^{69}$ It has been suggested that, even in the presence of severe thrombocytopenia, this procedure may be performed without prior platelet transfusion, provided that adequate surface pressure is applied. ${ }^{70}$

${ }^{\ddagger}$ Platelet count threshold will vary depending on the type and extent of surgery and presence of factors that may affect platelet function (eg, uremia, medications, extracorporeal circulatory support for surgery, comorbid conditions). ${ }^{69,70}$

CVC, central venous catheter; IVIG, intravenous immunoglobulin; LP, lumbar puncture.
}

ministration of immune-modulating therapies such as steroids or intravenous immunoglobulin may attenuate this process, resulting in a more rapid increase in platelet count. ${ }^{70}$ Patients with inherited and acquired platelet function abnormalities rarely require platelet transfusion. Alternative measures are proposed as first-line therapies in patients with platelet function defects who are actively bleeding or who require surgery (Table 4), except in cases of severe inherited disorders such as Glanzmann thrombasthenia or Bernard-Soulier syndrome. ${ }^{68}$ Human leukocyte antigen-matched platelets may be used in patients requiring multiple transfusions, given the possibility for alloimmunization. If human leukocyte antigen-matched platelets are not available, leukocyte-depleted concentrates may be used.

\section{Coagulation Factor Replacement}

For known or suspected coagulation factor deficiencies, factor replacement may be used to treat active bleeding or for hemostatic coverage before surgery. The indications for and necessity of factor replacement vary based on the specific bleeding disorder, the patient's bleeding tendency, the severity of bleeding, and, in the case of invasive procedures, the anticipated risk for bleeding. Because coagulation studies do not necessarily predict surgical bleeding risk in patients without an apparent bleeding disorder, the prophylactic use of factor replacement sources such as FFP simply to correct coagulation study abnormalities before surgery is inappropriate. ${ }^{26}$ In fact, prophylactic transfusion of FFP has not been shown to correct coagulation study abnormalities or reduce bleeding risk. ${ }^{26}$

Several options exist for replacing coagulation factors when indicated for treatment or prevention of bleeding $^{72-75}$ (Table 5). Given their wide availability, FFP and cryoprecipitate are mainstays of coagulation factor replacement worldwide. FFP is obtained from whole blood or by apheresis and contains all clotting factors. ${ }^{69}$ Cryoprecipitate is derived from thawing a single donor unit of FFP at $4^{\circ} \mathrm{C}$ and is rich in FVIII, VWF, FXIII, and fibrin- 
Table 5. Factor Replacement Sources

\begin{tabular}{|c|c|c|c|}
\hline Product & Composition & Indications & Comments \\
\hline \multirow[t]{4}{*}{ FFP } & \multirow[t]{4}{*}{ All coagulation factors } & $\begin{array}{l}\text { Multiple factor deficiencies (eg, in DIC, } \\
\text { liver disease) }\end{array}$ & $\begin{array}{l}\text { May be required in large volumes to } \\
\text { sufficiently boost levels of a single } \\
\text { coagulation factor }\end{array}$ \\
\hline & & $\begin{array}{l}\text { FV deficiency (may supplement with } \\
\text { platelet transfusions) }\end{array}$ & Not virally inactivated \\
\hline & & $\begin{array}{l}\text { FXI deficiency (in lieu of FXI } \\
\text { concentrate when there is a } \\
\text { heightened risk of thrombogenicity } \\
[\mathrm{eg} \text {, in the peripartum period }])^{70}\end{array}$ & \\
\hline & & $\begin{array}{l}\text { Other single-factor deficiencies when } \\
\text { single-factor concentrates or PCCs } \\
\text { are unavailable }\end{array}$ & \\
\hline \multirow[t]{2}{*}{ Cryoprecipitate } & \multirow{2}{*}{$\begin{array}{l}\text { Concentrate precipitated } \\
\text { from FFP; rich in FVIII, } \\
\text { VWF, FXIII, and } \\
\text { fibrinogen }^{72}\end{array}$} & \multirow[t]{2}{*}{ Used most often to replace fibrinogen ${ }^{72}$} & May be required in large volumes \\
\hline & & & Not virally inactivated \\
\hline \multirow[t]{3}{*}{ PCCs } & \multirow{3}{*}{$\begin{array}{l}\text { Highly purified } \\
\text { concentrates from pooled } \\
\text { normal plasma containing } \\
\text { FII, FIX, and FX } \\
( \pm \text { FVII })^{74,75}\end{array}$} & FII or FX deficiency & Virally inactivated ${ }^{74,75}$ \\
\hline & & $\begin{array}{l}\text { FIX or FVII* deficiencies when single- } \\
\text { factor concentrates are unavailable }\end{array}$ & $\begin{array}{l}\text { Contain known amounts of each } \\
\text { factor }^{74,75}\end{array}$ \\
\hline & & $\begin{array}{l}\text { 4-factor PCCs are indicated for } \\
\text { warfarin reversal }\end{array}$ & \\
\hline $\begin{array}{l}\text { Activated } \\
\text { PCCs }\end{array}$ & $\begin{array}{l}\text { PCC with FII, FIX, and FX } \\
\text { (mainly nonactivated) and } \\
\text { FVII (mainly in the } \\
\text { activated form) }\end{array}$ & Coagulation factor inhibitors ${ }^{\dagger}$ & \\
\hline \multirow[t]{11}{*}{$\begin{array}{l}\text { Single-factor } \\
\text { concentrates }\end{array}$} & Plasma derived & Respective single-factor deficiencies & $\begin{array}{l}\text { Treatment of choice for single- } \\
\text { factor deficiencies, when available }\end{array}$ \\
\hline & FVIII & \multirow{10}{*}{$\begin{array}{l}\text { rFVIIa is indicated as a bypassing agent } \\
\text { for FVIII and FIX inhibitors, }{ }^{\dagger} \text { as well } \\
\text { as for replacing FVII in FVII } \\
\text { deficiency }\end{array}$} & \multirow{10}{*}{$\begin{array}{l}\text { Risk for human viral contamination } \\
\text { is present but exceedingly low in } \\
\text { plasma-derived concentrates } \\
\text { because of screening and viral } \\
\text { inactivation procedures; it is } \\
\text { probably nonexistent in } \\
\text { recombinant concentrates }\end{array}$} \\
\hline & FVIII/VWF & & \\
\hline & FIX & & \\
\hline & FXIII & & \\
\hline & Fibrinogen & & \\
\hline & Recombinant & & \\
\hline & rFVIIa & & \\
\hline & rFVIII & & \\
\hline & rFIX & & \\
\hline & rFXIII & & \\
\hline
\end{tabular}

*Only 4-factor prothrombin complex concentrates (PCCs) may be used for replacement of factor VII (FVII); however, 4-factor PCCs are not universally available.

${ }^{\dagger}$ Although single-factor concentrates may be used for respective low-titer coagulation factor inhibitors (eg, plasma-derived or recombinant factor VIII [FVIII] for FVIII inhibitors), bypassing agents are generally recommended for active bleeding (for which they are considered first-line therapy) and for hemostatic coverage during invasive procedures in patients with inhibitors.

DIC, disseminated intravascular coagulation; FFP, fresh frozen plasma; F, factor; PCC, prothrombin complex concentrate; $\mathrm{rF}$, recombinant factor; VWF, von Willebrand factor.

ogen. ${ }^{72}$ FFP and cryoprecipitate are primarily indicated for replacement of multiple deficient clotting factors (eg, in DIC) and should be used only to replace single clotting factors when no specific concentrate is available. ${ }^{69,72}$ Large volumes of FFP are required to sufficiently boost a single coagulation factor, putting some patients at risk for fluid overload. In addition, despite substantial improvements in donor and plasma screening, transmission of blood-borne pathogens continues to be a theoreti- cal concern with FFP and cryoprecipitate because these products are not subjected to a specific viral inactivation process. $^{73}$ Therefore, virally inactivated FFP should be considered when available. ${ }^{73}$ For single-factor deficiencies, specific plasma-derived or recombinant factor concentrates are the treatment of choice, when available (Table 5). There are currently no single-factor concentrates for FII, FV, or FX. Prothrombin complex concentrates (PCCs), which are highly purified concen- 


\begin{tabular}{|c|c|}
\hline \multirow[t]{8}{*}{ Antifibrinolytics } & Include $\varepsilon$-aminocaproic acid and tranexamic acid \\
\hline & Interfere with the formation of the fibrinolytic enzyme plasmin from its precursor plasminogen \\
\hline & First-line therapy in rare disorders of fibrinolysis ( $\alpha 2$-antiplasmin and PAI- 1 deficiencies $)^{56}$ \\
\hline & $\begin{array}{l}\text { Systemic or topical antifibrinolytics may be used alone to treat or prevent mucosal bleeding in patients with } \\
\text { coagulation factor disorders, thus avoiding systemic hemostatic therapies }{ }^{1,66-68,73}\end{array}$ \\
\hline & Effective, first-line therapy for menorrhagia (with or without hormonal therapies) ${ }^{25,80,85}$ \\
\hline & $\begin{array}{l}\text { Tranexamic acid used anecdotally in patients with bleeding disorders, including as an adjunct to factor } \\
\text { replacement for major surgery in patients with hemophilia }{ }^{78,81,84} \text { and as a sole systemic hemostatic agent } \\
\text { primarily for minor procedures }\end{array}$ \\
\hline & $\begin{array}{l}\text { Also used for treatment of bleeding and for hemostatic coverage for minor surgery in patients with platelet } \\
\text { function disorders; may be used adjunctively with platelet transfusion and other nontransfusional } \\
\text { therapies in these patients }{ }^{83}\end{array}$ \\
\hline & $\begin{array}{l}\text { Theoretical risk for thrombogenesis; may be increased in patients who receive concurrent factor } \\
\text { replacement or who have certain bleeding disorders (eg, dysfibrinogenemia, in which antifibrinolytics are } \\
\text { contraindicated) }\end{array}$ \\
\hline \multirow[t]{5}{*}{ Desmopressin } & Transiently increases VWF and FVIII levels ${ }^{89}$ and augments platelet adhesiveness and aggregation ${ }^{68}$ \\
\hline & $\begin{array}{l}\text { Agent of choice for treatment or prevention of bleeding in most patients with type } 1 \text { VWD; however, } \\
\text { patients with type } 3 \text { VWD and most patients with type } 2 \text { VWD typically require factor concentrates } \\
\text { containing FVIII and VWF }\end{array}$ \\
\hline & $\begin{array}{l}\text { Other desmopressin-responsive bleeding disorders include mild hemophilia A and platelet function } \\
\text { disorders, in which the use of desmopressin for surgical coverage has been described }{ }^{21,87} \text {; also commonly } \\
\text { used for uremic bleeding }{ }^{88}\end{array}$ \\
\hline & Specifically used for treatment of menorrhagia in women with bleeding disorders ${ }^{25,80,91}$ \\
\hline & Patients, especially children, receiving desmopressin should be closely monitored for hyponatremia ${ }^{22,87}$ \\
\hline \multirow[t]{3}{*}{ Vitamin $\mathrm{K}$} & May be used in vitamin $\mathrm{K}$ deficiency and for bleeding caused by overmedication with warfarin \\
\hline & Widely available and inexpensive \\
\hline & Should be given intravenously or orally (not subcutaneously) for best absorption and fastest response $\mathrm{s}^{92-94}$ \\
\hline
\end{tabular}

FVIII, factor VIII; PAI, plasminogen activator inhibitor; VWD, von Willebrand disease; VWF, von Willebrand factor.

trates of specific coagulation factors (FII, factor IX, and FX \pm FVII) obtained from pooled normal plasma, should be used in lieu of FFP for deficiencies of FII or FX, when available, given that PCCs are virally inactivated and contain known amounts of each factor. ${ }^{74,75}$ Absent any other source, FFP is the only option for FV replacement; platelet transfusions may be considered as an additional source of $\mathrm{FV}$ in cases in which severe bleeding cannot be controlled with FFP alone. ${ }^{76}$

In cases of low factor levels due to an acquired inhibitor, factor replacement may be ineffective, except when the inhibitor titer (autoantibody burden) is very low. Low-titer inhibitors can sometimes be overcome by large amounts of factor. High-titer inhibitors, however, cannot be overcome with large doses of the decreased clotting factor; therefore, so-called bypassing agents are generally recommended for active bleeding (for which they are considered first-line therapy) and for hemostatic coverage during invasive procedures. An activated PCC product and a product containing recombinant activated FVII are the only 2 bypassing agents available. Although neither agent is as effective as replacing the missing factor (when factor replacement is an option), both have been shown to be helpful in achieving hemostasis in patients with coagulation factor inhibitors. ${ }^{77}$

\section{Nontransfusional Hemostatic Therapies}

Nontransfusional therapies such as antifibrinolytics ( $\varepsilon$-aminocaproic acid or tranexamic acid), $1,25,56,66-68,73,78-86$ desmopres$\sin ,{ }^{21,22,25,35,36,68,77,80,87-91}$ and vitamin $\mathrm{K}^{92-94}$ may be used for the treatment or prevention of bleeding in patients with bleeding disorders (Table 6) and occasionally as sole hemostatic agents for the treatment of mild mucosal bleeding or for hemostatic coverage during minor (eg, dental) procedures, thus sparing the patient exposure to transfusional therapies. Nontransfusional therapies can also be used as an adjunct to transfusional therapies in cases of more severe bleeding or for hemostatic coverage during major surgery.

Menorrhagia in women with bleeding disorders often is successfully managed with antifibrinolytics and hormone therapy, ${ }^{85}$ including combination or progestin-only contraceptives for maintenance ther- 
apy and conjugated estrogens for acute menorrhagia. ${ }^{25}$ Transfusional therapies may be used in refractory cases. ${ }^{25}$ Invasive options for controlling menorrhagia include the levonorgestrel-releasing intrauterine system, endometrial balloon tamponade, dilatation and curettage, and non-fertilitysparing procedures such as endometrial ablation, uterine artery embolization, and hysterectomy. ${ }^{25,91}$

\section{Conclusions}

Adults with undiagnosed bleeding disorders may initially present to their primary care provider with asymptomatic hematologic laboratory abnormalities or with abnormal bleeding symptoms in the outpatient or inpatient setting. Accordingly, family physicians should be prepared to recognize potential hallmarks of an underlying bleeding disorder and to initiate a proper workup and, in some cases, treatment, particularly if there is active bleeding. Consultation with a hematologist may be considered to assist with definitive diagnosis and, for patients who are ultimately diagnosed with a bleeding disorder, individualized long-term hemostatic management. The family physician serves as a crucial member of the multidisciplinary team providing care to adults with bleeding disorders and may play an especially important role in prioritizing routine and disease-related preventive care in this population.

The authors thank Lara Primak, MD (ETHOS Health Communications, Newtown, PA) for providing writing assistance.

\section{References}

1. Acharya SS, Coughlin A, Dimichele DM. Rare Bleeding Disorder Registry: deficiencies of factors II, V, VII, X, XIII, fibrinogen and dysfibrinogenemias. J Thromb Haemost 2004;2:248-56.

2. Wahlberg T, Blombäck M, Hall P, Axelsson G. Application of indicators, predictors and diagnostic indices in coagulation disorders. I. Evaluation of a self-administered questionnaire with binary questions. Methods Inf Med 1980;19:194-200.

3. Hassan AA, Kroll MH. Acquired disorders of platelet function. Hematology Am Soc Hematol Educ Program 2005:403-408.

4. Sadler JE, Rodeghiero F, ISTH SSC Subcommittee on von Willebrand Factor. Provisional criteria for the diagnosis of VWD type 1. J Thromb Haemost 2005;3:775-7.

5. Rodeghiero F, Tosetto A, Abshire T, et al. ISTH/ SSC bleeding assessment tool: a standardized questionnaire and a proposal for a new bleeding score for inherited bleeding disorders. J Thromb Haemost 2010;8:2063-5.

6. Tosetto A, Castaman G, Plug I, Rodeghiero F, Eikenboom J. Prospective evaluation of the clinical utility of quantitative bleeding severity assessment in patients referred for hemostatic evaluation. J Thromb Haemost 2011;9:1143-8.

7. ACOG Committee on Practice Bulletins-Gynecology. American College of Obstetricians and Gynecologists. ACOG practice bulletin: management of anovulatory bleeding. Int J Gynaecol Obstet 2001; 72:263-71.

8. Bolton-Maggs PH, Chalmers EA, Collins PW, et al. A review of inherited platelet disorders with guidelines for their management on behalf of the UKHCDO. Br J Haematol 2006;135:603-33.

9. Girolami A, Ruzzon E, Tezza F, Scandellari R, Vettore S, Girolami B. Arterial and venous thrombosis in rare congenital bleeding disorders: a critical review. Haemophilia 2006;12:345-51.

10. Inbal A, Muszbek L. Coagulation factor deficiencies and pregnancy loss. Semin Thromb Hemost 2003; 29:171-4.

11. Warner PE, Critchley HO, Lumsden MA, Campbell-Brown M, Douglas A, Murray GD. Menorrhagia I: measured blood loss, clinical features, and outcome in women with heavy periods: a survey with follow-up data. Am J Obstet Gynecol 2004;190: $1216-23$.

12. Romney G, Glick M. An updated concept of coagulation with clinical implications. J Am Dent Assoc 2009;140:567-74.

13. van Herrewegen F, Meijers JC, Peters M, van Ommen CH. Clinical practice: the bleeding child. Part II: disorders of secondary hemostasis and fibrinolysis. Eur J Pediatr 2012;171:201-14.

14. Van Ommen CH, Peters M. The bleeding child. Part I: primary hemostatic disorders. Eur J Pediatr 2012;171:1-10.

15. Cattaneo M. Inherited platelet-based bleeding disorders. J Thromb Haemost 2003;1:1628-36.

16. Cines DB, Bussel JB, McMillan RB, Zehnder JL. Congenital and acquired thrombocytopenia. Hematology Am Soc Hematol Educ Program 2005:390406.

17. Balduini CL, Pecci A, Noris P. Inherited thrombocytopenias. The evolving spectrum. Hamostaseologie 2012;32:259-70.

18. Noris P, Pecci A, Di Bari F, et al. Application of a diagnostic algorithm for inherited thrombocytopenias to 46 consecutive patients. Haematologica 2004; 89:1219-25.

19. Balduini CL, Cattaneo M, Fabris F, et al. Inherited thrombocytopenias: a proposed diagnostic algorithm from the Italian Gruppo di Studio delle Piastrine. Haematologica 2003;88:582-92. 
20. Konkle BA. Acquired disorders of platelet function. Hematology Am Soc Hematol Educ Program 2011: 391-6.

21. Coppola A, Di Minno G. Desmopressin in inherited disorders of platelet function. Haemophilia 2008; 14(Suppl 1):31-9.

22. Tuohy E, Litt E, Alikhan R. Treatment of patients with von Willebrand disease. J Blood Med 2011;2: 49-57.

23. Nichols WL, Hultin MB, James AH, et al. von Willebrand disease (VWD): evidence-based diagnosis and management guidelines, the National Heart, Lung, and Blood Institute (NHLBI) Expert Panel report (USA). Haemophilia 2008;14:171-232.

24. Lee CA, Chi C, Pavord SR, et al. The obstetric and gynaecological management of women with inherited bleeding disorders-review with guidelines produced by a taskforce of UK Haemophilia Centre Doctors' Organization. Haemophilia 2006;12:301-36.

25. James AH, Kouides PA, Abdul-Kadir R, et al. Evaluation and management of acute menorrhagia in women with and without underlying bleeding disorders: consensus from an international expert panel. Eur J Obstet Gynecol Reprod Biol 2011;158:124-34.

26. Holland L, Sarode R. Should plasma be transfused prophylactically before invasive procedures? Curr Opin Hematol 2006;13:447-51.

27. Bushick JB, Eisenberg JM, Kinman J, Cebul RD, Schwartz JS. Pursuit of abnormal coagulation screening tests generates modest hidden preoperative costs. J Gen Intern Med 1989;4:493-7.

28. Hayward CP, Moffat KA, Liu Y. Laboratory investigations for bleeding disorders. Semin Thromb Hemost 2012;38:742-52.

29. Kamal AH, Tefferi A, Pruthi RK. How to interpret and pursue an abnormal prothrombin time, activated partial thromboplastin time, and bleeding time in adults. Mayo Clin Proc 2007;82:864-73.

30. Green D. Interpreting coagulation assays. Blood Coagul Fibrinolysis. 2010;21(Suppl 1):S3-6.

31. Jones DW, Gallimore MJ, Mackie IJ, Harris SL, Winter M. Reduced factor XII levels in patients with the antiphospholipid syndrome are associated with antibodies to factor XII. Br J Haematol 2000;110: 721-6.

32. Mazodier K, Arnaud L, Mathian A, et al. Lupus anticoagulant-hypoprothrombinemia syndrome: report of 8 cases and review of the literature. Medicine (Baltimore) 2012;91:251-60.

33. Franchini M, Lippi G. Acquired factor VIII inhibitors. Blood 2008;112:250-5.

34. Knoebl P, Marco P, Baudo F, et al. Demographic and clinical data in acquired hemophilia A: results from the European Acquired Haemophilia Registry (EACH2). J Thromb Haemost 2012;10:622-31.

35. Collins P, Baudo F, Huth-Kuhne A, et al. Consensus recommendations for the diagnosis and treatment of acquired hemophilia A. BMC Res Notes 2010;3:161.
36. Huth-Kuhne A, Baudo F, Collins P, et al. International recommendations on the diagnosis and treatment of patients with acquired hemophilia A. Haematologica 2009;94:566-75.

37. Tay L, Duncan E, Singhal D, et al. Twelve years of experience of acquired hemophilia A: trials and tribulations in South Australia. Semin Thromb Hemost 2009;35:769-77.

38. Zeitler H, Ulrich-Merzenich G, Goldmann G, Vidovic N, Brackmann HH, Oldenburg J. The relevance of the bleeding severity in the treatment of acquired haemophilia - an update of a single-centre experience with 67 patients. Haemophilia 2010;16: 95-101.

39. Hay CRM, Negrier C, Ludlam CA. The treatment of bleeding in acquired haemophilia with recombinant factor VIIa: a multicentre study. Thromb Haemost 1997;78:1463-7.

40. Morrison AE, Ludlam CA, Kessler C. Use of porcine factor VIII in the treatment of patients with acquired hemophilia. Blood 1993;81:1513-20.

41. Sekhon SS, Roy V. Thrombocytopenia in adults: a practical approach to evaluation and management. South Med J 2006;99:491-8.

42. Drews RE. Critical issues in hematology: anemia, thrombocytopenia, coagulopathy, and blood product transfusions in critically ill patients. Clin Chest Med 2003;24:607-22.

43. McCrae KR. Thrombocytopenia in pregnancy. Hematology Am Soc Hematol Educ Program 2010: 397-402.

44. Abildgaard U, Heimdal K. Pathogenesis of the syndrome of hemolysis, elevated liver enzymes, and low platelet count (HELLP): a review. Eur J Obstet Gynecol Reprod Biol 2013;166:117-23.

45. Drews RE, Weinberger SE. Thrombocytopenic disorders in critically ill patients. Am J Respir Crit Care Med 2000;162(2 Pt 1):347-51.

46. Mannucci PM, Peyvandi F. TTP and ADAMTS13: when is testing appropriate? Hematology Am Soc Hematol Educ Program 2007:121-6.

47. Stasi R, Amadori S, Osborn J, Newland AC, Provan D. Long-term outcome of otherwise healthy individuals with incidentally discovered borderline thrombocytopenia. PLoS Med 2006;3:e24.

48. Arnold DM, Smith JW, Warkentin TE. Management of immune thrombocytopenia. In: Simon TL, Snyder EL, Stowell CP, Strauss RG, Solheim BG, Petrides M, eds. Rossi's principles of transfusion medicine. 4th ed. Oxford, UK: Blackwell Publishing Ltd; 2009. p. 344-75.

49. Peterson P, Hayes TE, Arkin CF, et al. The preoperative bleeding time test lacks clinical benefit: College of American Pathologists' and American Society of Clinical Pathologists' position article. Arch Surg 1998;133:134-9.

50. Castaman G, Tosetto A, Goodeve A, et al. The impact of bleeding history, von Willebrand factor 
and PFA-100(®) on the diagnosis of type 1 von Willebrand disease: results from the European study MCMDM-1VWD. Br J Haematol 2010;151: 245-51.

51. Sladky JL, Klima J, Grooms L, Kerlin BA, O’Brien SH. The PFA-100® does not predict delta-granule platelet storage pool deficiencies. Haemophilia 2012; 18:626-9.

52. Akin M, Polat Y. Platelet function analyser (PFA)100(®) closure time in the evaluation of non-steroidal anti-inflammatory drug-induced platelet dysfunction in children with bleeding symptoms. Blood Transfus 2012;10:545-6.

53. Jámbor C, von Pape KW, Spannagl M, Dietrich $W$, Giebl A, Weisser H. Multiple electrode whole blood aggregometry, PFA-100, and in vivo bleeding time for the point-of-care assessment of aspirin-induced platelet dysfunction in the preoperative setting. Anesth Analg 2011;113:31-9.

54. Sokkary NA, Venkateswaran L, Dietrich JE, Teruya J. Platelet function disorders and menorrhagia in adolescents: a review of laboratory diagnosis. J Pediatri Adolesc Gynecol 2012;25:233-7.

55. Favaloro EJ. The utility of the PFA-100 in the identification of von Willebrand disease: a concise review. Semin Thromb Hemost 2006;32:537-45.

56. Brown DL. Congenital bleeding disorders. Curr Probl Pediatr Adolesc Health Care 2005;35:38-62.

57. Carpenter SL, Mathew P. Alpha2-antiplasmin and its deficiency: fibrinolysis out of balance. Haemophilia 2008;14:1250-4.

58. Jackson SC, Odiaman L, Card RT, van der Bom JG, Poon MC. Suspected collagen disorders in the bleeding disorder clinic: a case-control study. Haemophilia 2013;19:246-50.

59. Karimi M, Bereczky Z, Cohan N, Muszbek L. Factor XIII deficiency. Semin Thromb Hemost 2009; 35:426-38.

60. Mehta R, Shapiro AD. Plasminogen activator inhibitor type 1 deficiency. Haemophilia 2008;14: 1255-60.

61. Daniele F, Rossi V, Santoro C. Effective management of intracranial haemorrhage with continuous infusion of highly purified von Willebrand factor/ factor VIII complex concentrate in an adult with severe haemophilia A. Blood Transfus 2011;9:472-4.

62. Kalsi H, Nanayakkara L, Pasi KJ, Bowles L, Hart DP. Access to primary dental care for patients with inherited bleeding disorders. Haemophilia 2012;18: 510-5.

63. Kumar JN, Kumar RA, Varadarajan R, Sharma N. Specialty dentistry for the hemophiliac: is there a protocol in place? Indian J Dent Res 2007;18:48-54.

64. Fogarty PF, Kouides P. How we treat: patients with haemophilia undergoing screening colonoscopy. Haemophilia 2010;16:363-5.

65. Brewer A, Correa ME. Guidelines for dental treatment of patients with inherited bleeding disorders.
Montreal, Quebec, Canada: World Federation of Hemophilia Dental Committee; 2006. Available from: http://www1.wfh.org/publication/files/pdf1190.pdf. Accessed May 16, 2014.

66. Hewson I, Makhmalbaf P, Street A, McCarthy P, Walsh M. Dental surgery with minimal factor support in the inherited bleeding disorder population at the Alfred Hospital. Haemophilia 2011;17:e185-8.

67. Lee AP, Boyle CA, Savidge GF, Fiske J. Effectiveness in controlling haemorrhage after dental scaling in people with haemophilia by using tranexamic acid mouthwash. Br Dent J 2005;198:33-8, discussion 26.

68. Seligsohn U. Treatment of inherited platelet disorders. Haemophilia 2012;18(Suppl 4):161-5.

69. Liumbruno G, Bennardello F, Lattanzio A, Piccoli P, Rossetti G; Italian Society of Transfusion Medicine and Immunohaematology (SIMTI) Work Group. Recommendations for the transfusion of plasma and platelets. Blood Transfus 2009;7:132-50.

70. British Committee for Standards in Haematology, Blood Transfusion Task Force. Guidelines for the use of platelet transfusions. Br J Haematol 2003;122: $10-23$.

71. Lisman T, Porte RJ. Rebalanced hemostasis in patients with liver disease: evidence and clinical consequences. Blood 2010;116:878-85.

72. O'Shaughnessy DF, Atterbury C, Bolton Maggs P, et al. Guidelines for the use of fresh-frozen plasma, cryoprecipitate and cryosupernatant. Br J Haematol 2004;126:11-28.

73. Peyvandi F, Palla R, Menegatti M, Mannucci PM. Introduction. Rare bleeding disorders: general aspects of clinical features, diagnosis, and management. Semin Thromb Hemost 2009;35:349-55.

74. Rodgers GM. Prothrombin complex concentrates in emergency bleeding disorders. Am J Hematol 2012; 87:898-902.

75. United Kingdom Haemophilia Centre Doctors' Organisation. Guidelines on the selection and use of therapeutic products to treat haemophilia and other hereditary bleeding disorders. Haemophilia 2003;9: 1-23.

76. Bolton-Maggs PH, Perry DJ, Chalmers EA, et al. The rare coagulation disorders-review with guidelines for management from the United Kingdom Haemophilia Centre Doctors' Organisation. Haemophilia 2004;10:593-628.

77. Baudo F, Collins P, Huth-Kühne A, et al. Management of bleeding in acquired hemophilia A: results from the European Acquired Haemophilia (EACH2) Registry. Blood 2012;120:39-46.

78. De Bels D, Demeere JL, Dugauqier J, Louis P, Van der Vorst E. Continuous infusion of factor VIIIc during heart surgery in a patient with haemophilia A. Eur J Anaesthesiol 2004;21:984-6.

79. Ker K, Edwards P, Perel P, Shakur H, Roberts I. Effect of tranexamic acid on surgical bleeding: sys- 
tematic review and cumulative meta-analysis. BMJ 2012;344:e3054.

80. Kouides PA, Byams VR, Philipp CS, et al. Multisite management study of menorrhagia with abnormal laboratory haemostasis: a prospective crossover study of intranasal desmopressin and oral tranexamic acid. Br J Haematol 2009;145:212-20.

81. Krakow EF, Walker I, Lamy A, Anderson JA. Cardiac surgery in patients with haemophilia B: a case report and review of the literature. Haemophilia 2009;15:108-13.

82. Lin PC, Hsu CH, Huang CC, Chen WS, Wang JW. The blood-saving effect of tranexamic acid in minimally invasive total knee replacement: is an additional pre-operative injection effective? J Bone Joint Surg Br 2012;94:932-6.

83. Nurden AT, Freson K, Seligsohn U. Inherited platelet disorders. Haemophilia 2012;18(Suppl 4): 154-60.

84. Tang M, Wierup P, Terp K, Ingerslev J, Sorensen B. Cardiac surgery in patients with haemophilia. Haemophilia 2009;15:101-7.

85. Venkateswaran L, Yee DL. Rare bleeding disorders in young women. J Pediatr Adolesc Gynecol 2010; 23(6 Suppl):S38-42.

86. Yang ZG, Chen WP, Wu LD. Effectiveness and safety of tranexamic acid in reducing blood loss in total knee arthroplasty: a meta-analysis. J Bone Joint Surg Br 2012;94:1153-9.

87. Dunn AL, Cox Gill J. Adenotonsillectomy in patients with desmopressin responsive mild bleeding disorders: a review of the literature. Haemophilia 2010;16:711-6.

88. Hedges SJ, Dehoney SB, Hooper JS, Amanzadeh J, Busti AJ. Evidence-based treatment recommendations for uremic bleeding. Nat Clin Pract Nephrol 2007;3:138-53.

89. Mannucci PM. Desmopressin (DDAVP) in the treatment of bleeding disorders: the first 20 years. Blood 1997;90:2515-21.

90. Mannucci PM. How I treat patients with von Willebrand disease. Blood 2001;97:1915-9.

91. Sanders S, Purcell S, Silva M, Palerme S, James P. Relationship between diagnosis and intervention in women with inherited bleeding disorders and menorrhagia. Haemophilia 2012;18:e273-6.

92. Crowther MA, Douketis JD, Schnurr T, et al. Oral vitamin $\mathrm{K}$ lowers the international normalized ratio more rapidly than subcutaneous vitamin $\mathrm{K}$ in the treatment of warfarin-associated coagulopathy. A randomized, controlled trial. Ann Intern Med 2002; 137:251-4.

93. Lubetsky A, Yonath H, Olchovsky D, Loebstein R, Halkin H, Ezra D. Comparison of oral vs intravenous phytonadione (vitamin K1) in patients with excessive anticoagulation: a prospective randomized controlled study. Arch Intern Med 2003;163: 2469-73.

94. Raj G, Kumar R, McKinney WP. Time course of reversal of anticoagulant effect of warfarin by intravenous and subcutaneous phytonadione. Arch Intern Med 1999;159:2721-4. 